\title{
A Case Study of Direct Current Resistivity Method for Disaster Water Source Detection in Coal Mining
}

\author{
Yangzhou Wang, ${ }^{1,2}$, Jingcun Yu ${ }^{1}$, Xihui Feng ${ }^{3}, \mathrm{Li} \mathrm{Ma}^{3}$, Qianhui Gao, ${ }^{1,}$, Benyu Su ${ }^{1,3}$, Xiuju Xing ${ }^{4}$ \\ ${ }^{1}$ China University of Mining and Technology, \\ Xuzhou, Jiangsu Province 221116, China \\ ${ }^{2}$ Shandong Energy Group South America Co., Ltd, \\ Qingdao, Shandong Province 266000, China \\ ${ }^{3}$ Key Laboratory of Coal Resources Exploration and Comprehensive Utilization, Ministry of Natural \\ Resources, \\ $X i$ 'an 710021, China \\ ${ }^{4}$ China Coal Science and engineering Xi'an Research Co., Ltd, \\ $X i$ 'an 710077, China \\ huixinxin@126.com
}

\begin{abstract}
Disaster water sources in underground coal seam always danger mining safety. The current electromagnetic methods are not effective to detect the disaster water sources in the underground due to noise interference generated by large metal equipment. To address this problem, the direct current resistivity method is proposed to detect the disaster water sources in the underground coal seams. The relationship between the resistivity distribution and water faults was investigated, and we found that a low resistivity distribution indicates a disaster water source. To verify the proposed method, both numerical simulations and field test have been carried out and the analysis results show that the low resistivity distribution can be used to correctly detect the disaster water sources. Most importantly, the proposed method has detected three threats of mine water disaster in the field test in a real coal seam in Lan County coal mine, China. As a result, the present work provides an important and solid support to coal mining safety.
\end{abstract}

Index Terms-Coal mining safety; Mine direct current resistivity method; Mine water disaster; Coal mine floor.

\section{INTRODUCTION}

During the underground coal mining process, the earth

Manuscript received 17 October, 2020; accepted 15 March, 2021.

This research was funded by the Fundamental Research Funds for the Central Universities, CUMT under Grant No. 2017WA02, by the National Natural Science Foundation under Grant No. 41974151, by the Jiangsu province Natural Science Foundation under Grant No. BK20181360, by the Major Scientific and Technological Innovation Project of Shandong Province of China under Grant No. 2019JZZY010820, by the National Key Research and Development Program of China under Grant No. 2018YFC0807804-02, by the Open Fund of Shaanxi Key Laboratory of Geological Support for Coal Green Exploitation under Grant No. DZBZ2020-06, by the Shaanxi Province Technology Innovation Guidance under Grant No. 2020CGHJ-005, by the Open Fund of Key Laboratory of Coal Resources and Exploration and Comprehensive Utilization, Ministry of Natural Resources the under Grant No. KF2021-6, and by the Major Scientific and Technological Innovation Projects in Shandong Province under Grant No. 2019SDZY010101. stress will be acted on the coal wall [1]. At this moment, it is very possible that the coal roof water and floor confined water will break into the coal mining tunnel, resulting in severe disasters [2]. Hence, it is crucial to detect the disaster water sources to prevent unexpected mining accidents. As a result, geophysical mine prospecting technologies for predicting geological anomalous bodies with bearing water have attracted increasing attention. Among these geophysical methods, mine resistivity methods and mine transient electromagnetic method have been widely used because of their convenient construction [3] and strong adaptability to limited underground space [4]. The most popular method for detecting disaster water sources is the transient electromagnetic method [5]. However, due to the large metal equipment in the coal tunnel, the noise generated by the metal equipment will contaminate the electromagnetic information [6]. As a result, the transient electromagnetic method [7] is not always applicable to coal mining.

Compared with the transient electromagnetic method, the direct current resistivity method theory of field distribution is rather simple and it can overcome the electromagnetic noise problem generated from metal equipment by employing electrodes to transmit and record the signal [8]. Although multielectrode systems and multicore cable are not easy to be carried in the tunnel, the application effect should come first Although there are 20 years since the direct current resistivity method has been used in the coal mine tunnel for predicting geological hazards, many application cases are very simple and use only the apparent resistivity curve to express the distribution of resistivity, such as direct current vertical resistivity sounding. However, unlike the ground surface circumstances for direct current resistivity methods on the surface, it is applied in the tunnel and the influence of the roadway on the distribution of the physical field is a special and inherent problem for mine-based geophysical methods, creating a difficult challenge for measurement, data 
processing, and geological interpretation [9]. Hence, to our best knowledge, the 3D resistivity inversion method professionally used for data processing and interpretation for direct current resistivity data collected in the coal mine tunnel has not been found in the detection of disaster water sources in the literature yet.

As well known that the key issues of data processing are the algorithms of forward and inversion. The comprehensive theory of DC resistivity is given in geophysical textbooks, such as Reynolds [10] and Parasnis [11]. The purpose of the forward modelling is to study the distribution of electrical field in the earth by numerical simulation. Finite difference method [12] and finite element [13] method are employed to subdivision mesh. Recently, unstructured tetrahedral meshes are very popular due to their function of efficient local mesh refinement and the most flexible description of arbitrary model geometry. Hence, the most popular is the mesh technique [14]. In addition, high efficient solver is necessary for computing large matrix [15]. Compared with preconditioned conjugate gradient method for solving large matrix, multifrontal direct solvers can automatically search the most effective iteration direction [16]. Hence, both mesh technique and computation solvers are crucial factors for the data processing algorithms.

The motivation of this work is to evaluate the direct current resistivity method in detecting the disaster water sources in the coal tunnel. Based on the electrical resistivity differences between the target and surrounding rock, the influence of the electrical field due to the geological anomalous bodies was investigated. The electrical potential from the artificially established stable current field was observed and the recorded signals were analysed to recognize the distribution of underground anomalous bodies (such as the water-bearing structure, collapse column, and fault fissure). The analysis result demonstrates that the large resistivity difference between the geological anomalous bodies and the surrounding coal rock can be detected by the direct current resistivity method. Hence, the direct current resistivity method can effectively explore these geological anomalous bodies in the coal mining process.

\section{FIELD WORK INFORMATION}

The coal mine of interest is located in Lan County, Luliang City, Shanxi Province, China, as shown in Fig. 1. Shanxi is one of the provinces with large coal reserves in China. Besides, it has a wide distribution of coal and the quality of the coal is excellent. Hence, it plays a pivotal role in China economic construction. The research area is located in the northwest of China. Although the ground climate is relatively dry, the underground water is quite abundant [17], especially below 200 meters, which brings a great threat to the coal mining. Therefore, coal mining companies attach great importance to mine water hazard prevention and they are also very willing to invest research funds in mine water hazard prevention.

Hence, the purpose of this research is to determine the distribution of rich water areas, as well as the relationship between the rich water areas and the fault fissures. As shown in Fig. 1, the area enclosed by the blue lines is the working face. It has been verified that there are many fault fissures in the working face; especially, there are two big fault fissures through the half working face. It is very dangerous that the water bearing area is very possible to be conducted into the tunnel. Hence, we have to detect the water yield property and fault condition before mining. As mentioned above, the direct current resistivity method will be employed to calculate the resistivity distribution of the earth, while the water bearing area can be determined by the lower resistivity relative to the surrounding rock.

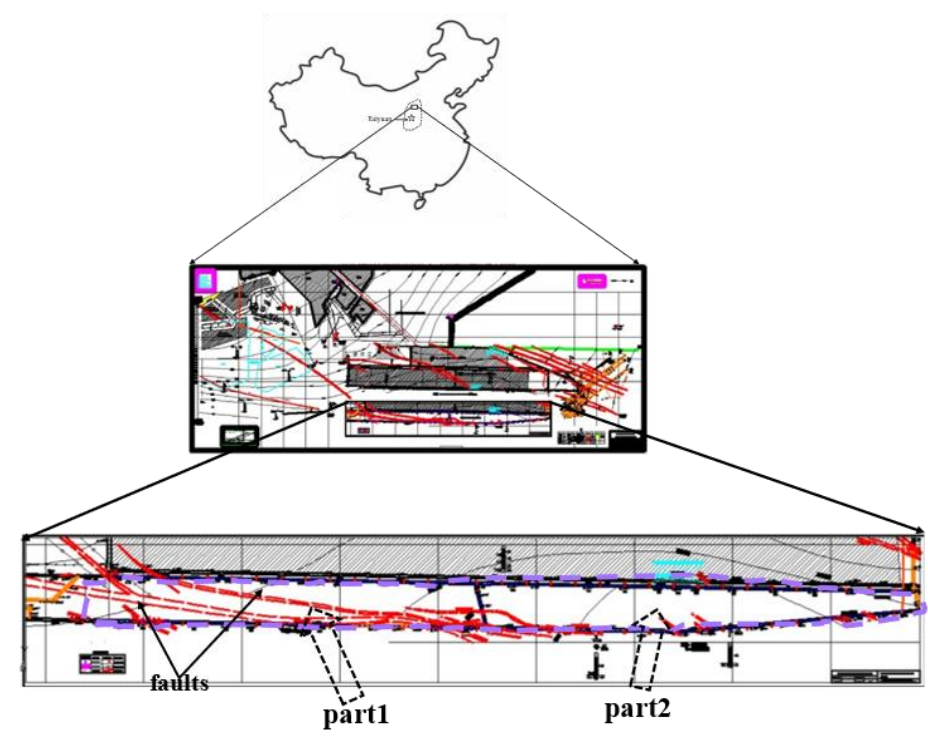

Fig. 1. Location of field work and coal mine geology.

To explore the geological information of the working face in detail, we separate the whole working face into two parts, as shown in Fig. 1. We designed the electrode arrangement of "U" shape by using one hundred and sixty electrodes for Part 1 and hundred and sixty-five electrodes for Part 2 to close the research area. The purpose of the design is to make the current cover of the entire research area to enhance detecting ability and resolution. The layout style of the electrodes of the first part and the second part is shown in Fig. 2. and Fig. 3 , respectively. 


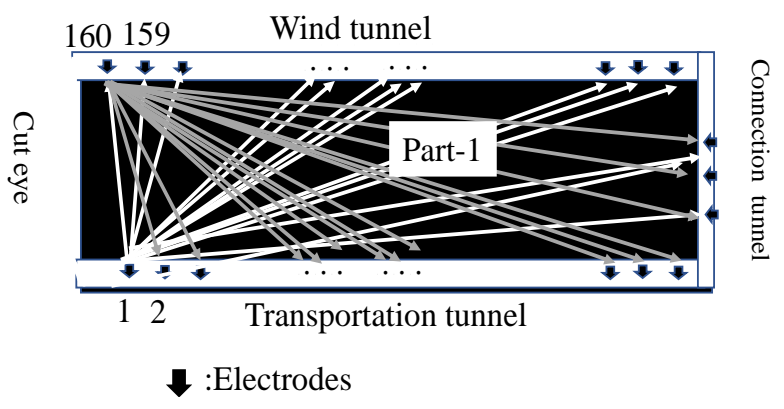

Fig. 2. Distribution of electrodes to collect data for exploring floor for Part 1. The white lines indicate that No. 1 electrode is a transmitter and the rest electrodes are receivers, while the grey lines show that No. 160 electrode is a transmitter and the rest electrodes are receivers. In fact, besides No. 1 and No. 160 , the other electrodes also can be transmitters and receivers.

In the field work, the data are collected by mine resistivity equipment, which can achieve the task that one pair of electrodes to be the transmitter and the remaining electrodes to be receivers for each time measuring. The other advantage of mine resistivity equipment is that the whole electrical field can be completely recorded and greatly enhance the efficiency of collecting data in the coal mine tunnel. Besides, the mine resistivity equipment is shown in Fig. 4.

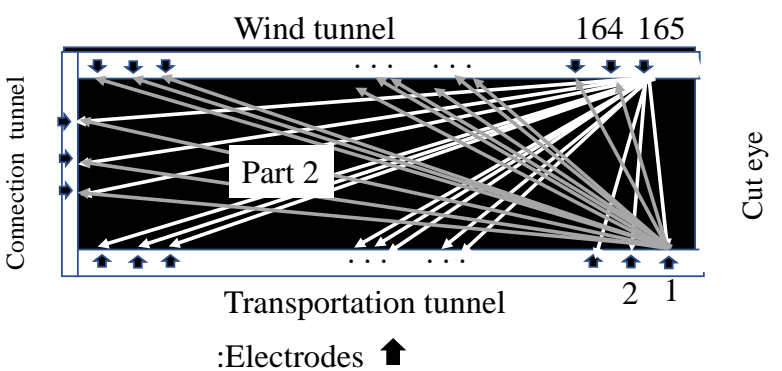

Fig. 3. Distribution of electrodes to collect data for exploring floor for Part 2. The grey lines indicate that No. 1 electrode is a transmitter and the rest electrodes are the receivers, while the white lines show that No. 165 electrode is a transmitter and the rest electrodes are the receivers. In fact, besides No. 1 and No. 165, the other electrodes also can be transmitters.

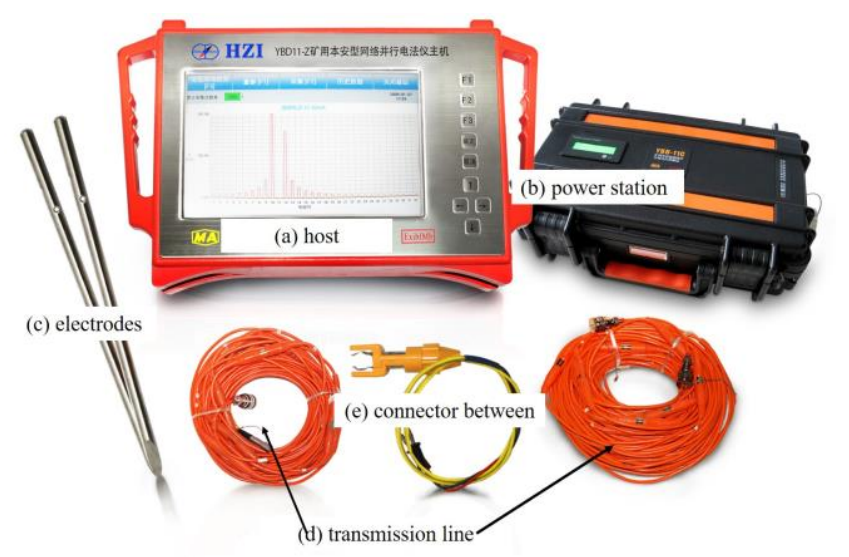

Fig. 4. Mine direct resistivity equipment: (a) host, (b) power station, (c) electrodes, and (d) transmission line.Problems of Forward and Inversion.

The relationship between the electrical potential and the geoelectrical parameters can be described by the Poisson's equation, as shown in (1) [18]

$$
\nabla \times(-\sigma \nabla \varphi)=I\left(\delta\left(r-r_{s+}\right)-\delta\left(r-r_{s-}\right)\right)
$$

where $\sigma$ is the conductivity structure of a medium, $\varphi$ is the electrical potential field induced by a dipole, and $I$ is the electrical current from a dipole. The dipole is represented by two delta functions centred on the positive and negative source locations ( $r_{s+}$ and $\left.r_{s-}\right)$.

To compute the solution of (1), the system is discretized using the finite volume method (FVM) [19]. The boundary conditions are embedded in the discrete differential operators. As a result, (1) can be described using (2)

$$
A \varphi=-q,
$$

where $A=\operatorname{Div}\left(\operatorname{diag}\left(A_{c}^{f^{T}} \sigma^{-1}\right)\right)^{-1} ; \quad A_{c}^{f^{T}}$ is related with the mesh size and $\sigma^{-1}$ is related with the conductivity of the meshing element. Finally, the solution of $\varphi$ can be computed by solving this linear equation [20].

Direct current resistivity inverse problem may be considered as a mirror from the space of potential data to the space of resistivity model [21].

The constructed objective function can be described by the following (3) [21]

$$
\phi(m)=\sum_{i=1}^{n}\left\|W^{(i)}\left(d^{(i)}(m)-d_{\text {obs }}^{(i)}\right)\right\|_{2}^{2}+\frac{\beta}{2}\left\|G w\left(m-m_{r e f}\right)\right\|_{2}^{2},
$$

where $W(i)$ is a diagonal weighting matrix that is multiplied by the $i_{\text {th }}$ residual vector

$$
W^{(i)}=\operatorname{diag}\left(\frac{1}{\left|d_{o b s}^{(i)}\right| \times S D\left(d_{o b s}^{(i)}\right)+\varepsilon}\right), \quad i=1,2, \cdots, n,
$$

where $S D\left(d_{\text {obs }}^{(i)}\right)$ is the element-wise standard deviation of each data, $\varepsilon$ is a small positive constant that ensures a cut-off value, so an extremely large weight is not given to very low amplitude data, and $d_{o b s}^{(i)}$ is the observed data.

By solving (3) using the minimization method, it yields a model update parameter, $\partial p$, which will be incorporated into the current model by

$$
p_{k+1}=p_{k}+\beta_{k} \partial p
$$

where $\beta_{k}$ is the current line search parameter, $p_{k}$ is the current model, and $p_{k+1}$ is the new model. The line search parameter is used to control the magnitude of the update.

\section{NUMERICAL SIMULATION}

To verify the correctness of the algorithm, we compared with the analytical solution and the numerical solution. There is an analytical solution for uniform earth medium and the analytical solution can be computed by (6) [22]

$$
\mathbf{u}=\frac{\rho \mathbf{I}}{4 \pi}\left[\frac{1}{\sqrt{\left(\mathbf{D}-\mathbf{z}_{\mathbf{p}}\right)^{2}+\left(\mathbf{r}_{\mathbf{p}}\right)^{2}}}+\frac{1}{\sqrt{\left(\mathbf{D}+\mathbf{z}_{\mathbf{p}}\right)^{2}+\left(\mathbf{r}_{\mathbf{p}}\right)^{2}}}\right],
$$

where $\rho$ is the resistivity of the earth, D is the depth of the earth, $\mathbf{z}_{\mathbf{p}}$ is the depth of the measurement point, and $\mathbf{r}_{\mathbf{p}}$ is the 
radial distance from the measurement point to source.

It is assumed that the resistivity of the earth is $100 \mathrm{ohm}-\mathrm{m}$ and the coordinates of the source are located on $(0,0,80 \mathrm{~m})$. Besides, the measurement line is parallel to the $\mathrm{X}$-axis and the distance between $\mathrm{X}$-axis and the measurement line is $1 \mathrm{~km}$, as shown in Fig. 5. The comparison between the numerical solution and analytical solution is shown in Fig. 6. Numerical solution and analytical solution match very well which shows that the algorithm is correct.

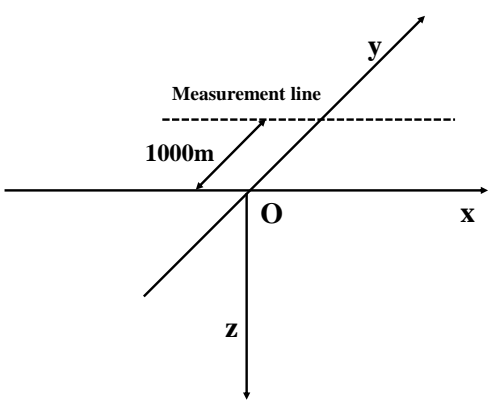

Fig. 5. Schematic diagram of line layout.

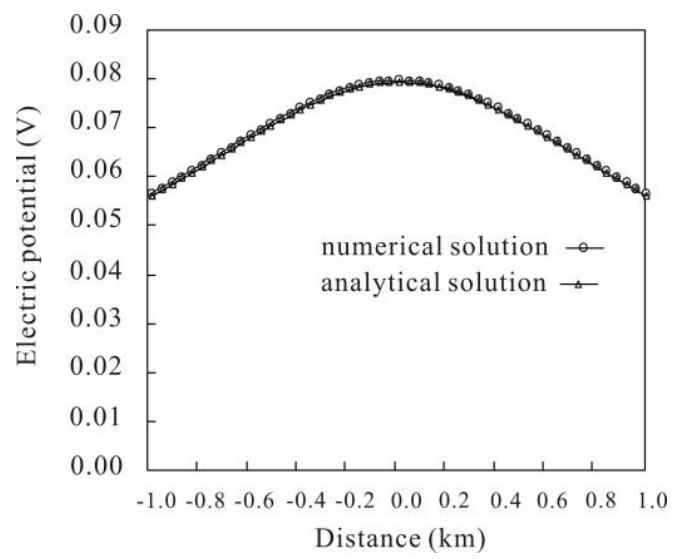

Fig. 6. Comparison between numerical solution and analytical solution.

In the actual detection process in the coal mining tunnel, the geology of the floor is critical for the detection performance. Therefore, the current flow into the floor sandstone by the electrodes was considered. The numerical simulation model is shown in Fig. 7.

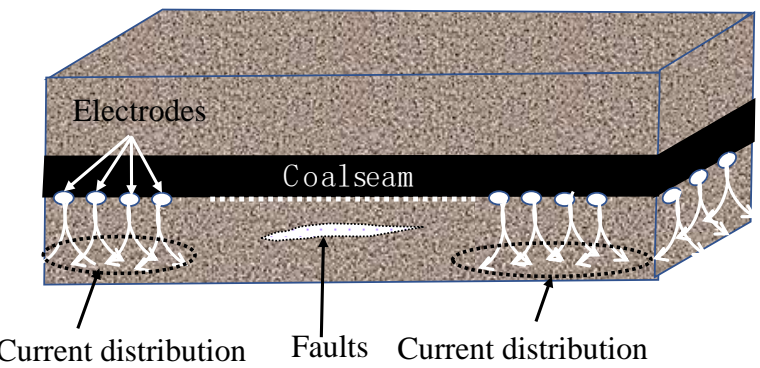

Fig. 7. Schematic diagram of coal seam floor detection by direct current resistivity.

To perform the numerical simulation, a mining geological model was established in Fig. 8. One hundred and sixty-five electrodes were used to measure the electrical potential in the tunnel. An anomalous geological body was inserted with rich water to simulate the water fault (see Fig. 8).

To exactly compute the solution, the tetrahedral element [23] is employed to mesh the geological model as shown in Fig. 9, which is shown by Paraview software [24] to show mesh model and modelling results. Besides, the natural boundary conditional is adopted to solve the boundary problems, as well as enough large distance from the research area to the boundary is made (see Fig. 9). In fact, only the small black area is the target area.

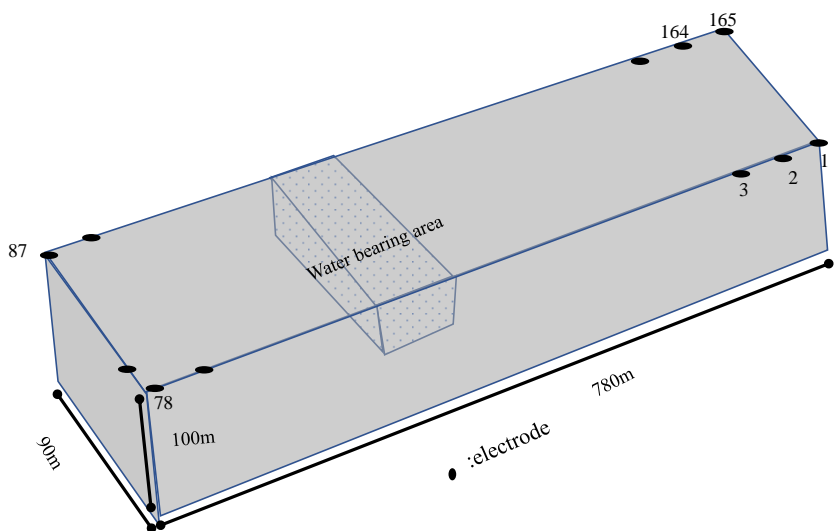

Fig. 8. Numerical geological model for numerical simulation.

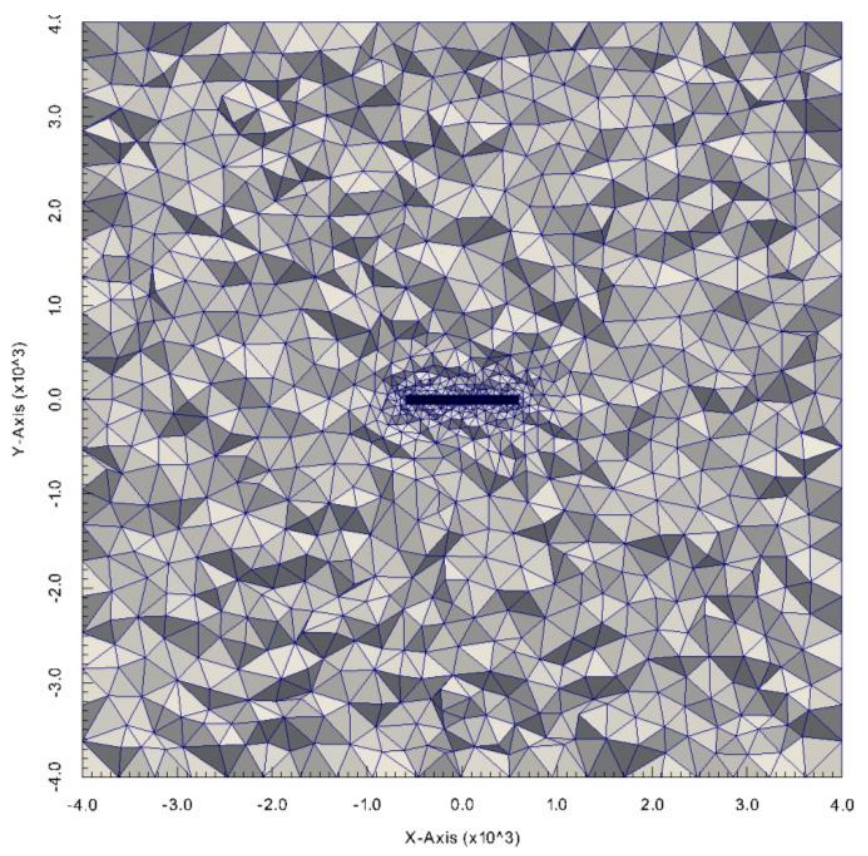

Fig. 9. Meshing results of the numerical geological model.

Figure 10 shows the inversion results, which are compared with the forward model as shown in Fig. 8. As can be seen, they match very well with each other. Besides, the modelling results are really $3 \mathrm{D}$ data volume and any slice can be cut to show geological information in detail. Actually, in Fig. 10, the slice of inversion results in the direction of $y=50 \mathrm{~m}$ is shown. In Fig. 11, the detailed information of the earth is exhibited, and we can clearly see the distribution of low resistivity.

\section{FIELD WORK}

According to the actual mining geological information, as described in Fig. 1, a field test has been conducted to verify the proposed method. The geological model of Part 1 was established in Fig. 12 and one hundred and sixty electrodes were used in the real coal mine tunnel to collect the electrical potential. As mentioned above, one pair of electrodes to be the transmitter and the remaining electrodes to be receivers for each time measuring. 


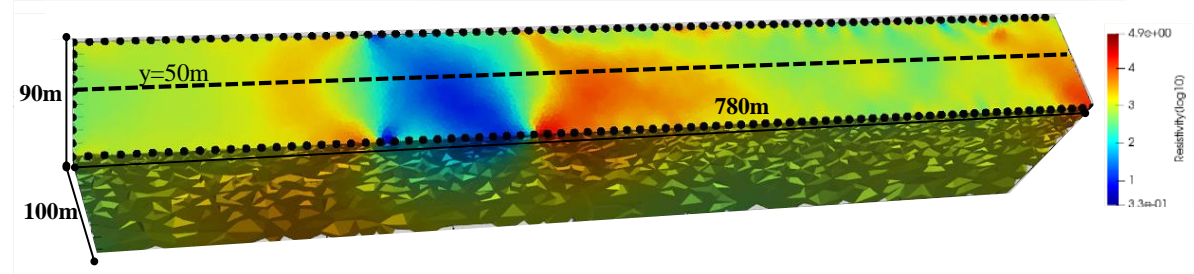

Fig. 10. Inversion results of the numerical geological model of Fig. 8 .

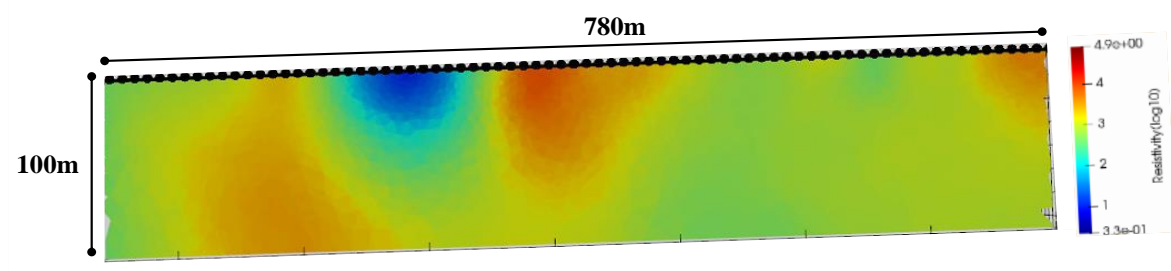

Fig. 11. The slice of the inversion results as described in Fig. 10.

Hence, massive data can be obtained by combination between different electrodes among one hundred and sixty electrodes. However, we only extract 4500 data by measurement style of ANM [25]. Measurement current is shown in Fig. 12, which is the electrical current, and we can clearly see the shape of the transmit waveform of the bipolar square wave. In Fig. 13, the difference of the voltages between the electrodes of $M$ and $N$ in the measurement style of $A M N$ is shown.

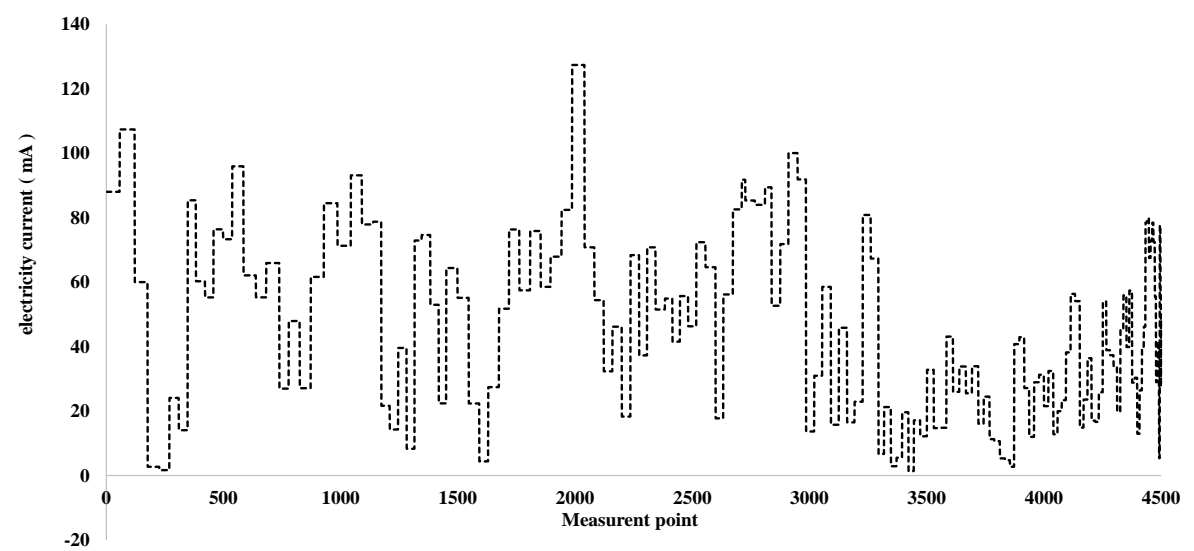

Fig. 12. The data of measurement current.

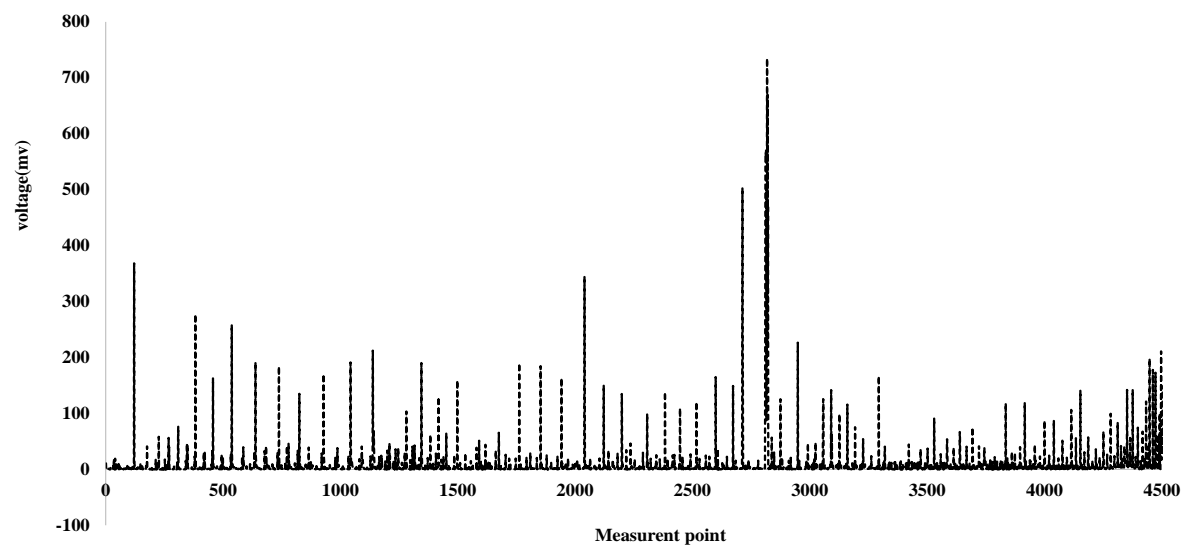

Fig. 13. The distribution of measurement electrical voltage.

Based on the electrical current information and electrical voltage, the inversion jobs have been done. Firstly, according to the geological information, the geological model has been built and the initial model is regarded to be homogeneous, as shown in Fig. 14. In Fig. 15, the inversion results from the collected electrical information (Fig. 12) by inversion method are shown. As shown in Fig. 15, three areas with low resistivity were observed. As mentioned above, these areas with low resistivity are potential disaster sources and it is very possible to cause large threat for coal mining. To examine the detailed geological information of these areas, the slice in the middle of $\mathrm{Y}$-axis has been cut and it is shown in Fig. 16. As can be clearly seen, the occurrence of these three low resistivity areas in the geological anomalous bodies was observed. Moreover, the low resistivity geological anomalous bodies in No. 2 in the figure were very possible to 
conduct deep limestone water into the coal tunnel due to its shape of resistivity distribution.

To explore Part 2, one hundred and sixty-five electrodes have been employed to collected data and totally 4040 data have been collected. Thereinto, in Fig. 17, the electrical current values are presented, while in Fig. 18, the voltage between $M$ and $N$ is shown.

To obtain the resistivity distribution of the Part 2 area by inversion, according to the known geological information, the homogeneous geological model has been built to be as an initial model, as shown in Fig. 19.
Based on the measurement data and inversion algorithm, the inversion results can be obtained, as shown in Fig. 20. With the purpose of knowing the detailed information of Part 2, the slice is cut from the 3D data volume, as shown in Fig. 21. In Fig. 20 and Fig. 21, no low resistivity information is exhibited, which means that there is no mining water disaster threat in the Part 2 area. However, three high resistivity anomalous bodies are possible to be collapse columns and they are also important geological information for coal mining.

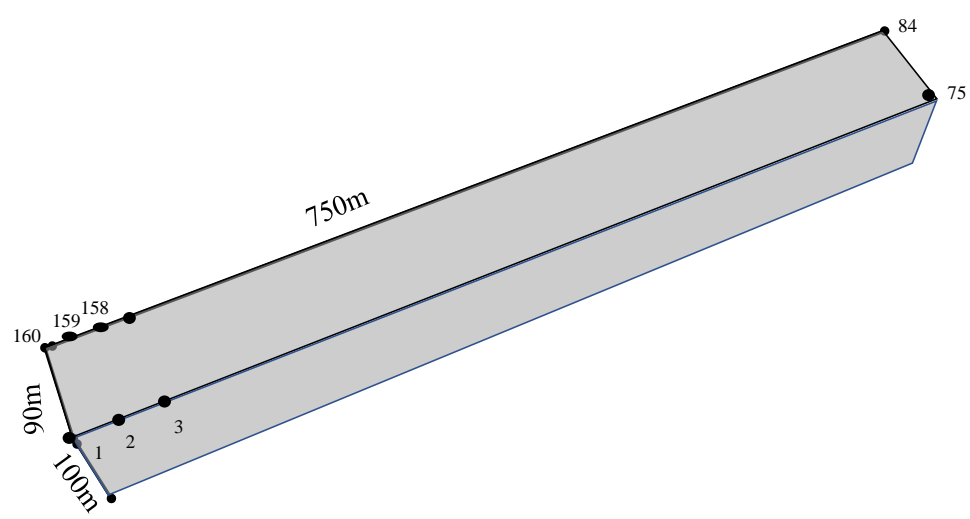

Fig. 14. The geological model of Part 1.

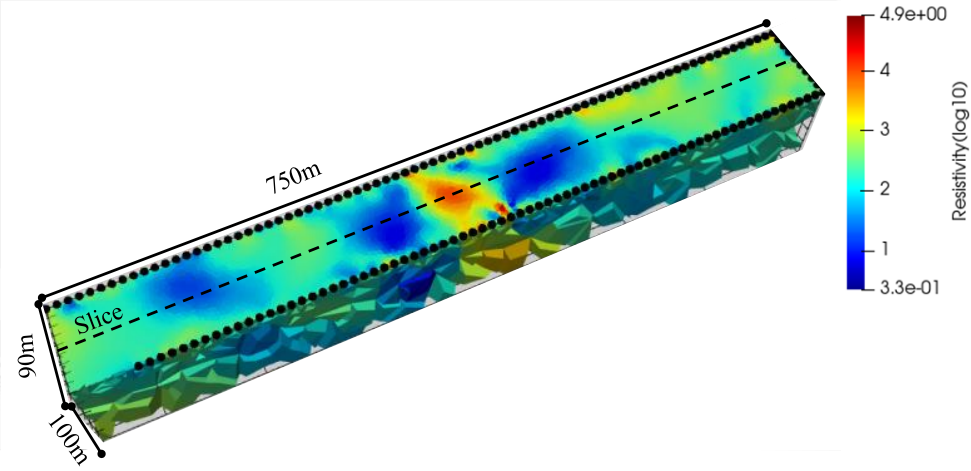

Fig. 15. Inversion results from the collected electrical potential of Part 1.

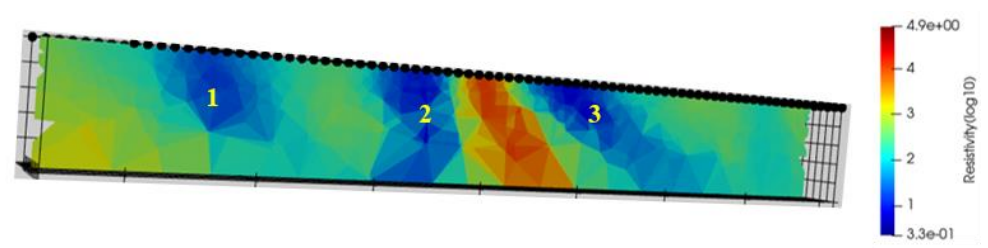

Fig. 16. The slice of the inversion results as described in Fig. 8 .

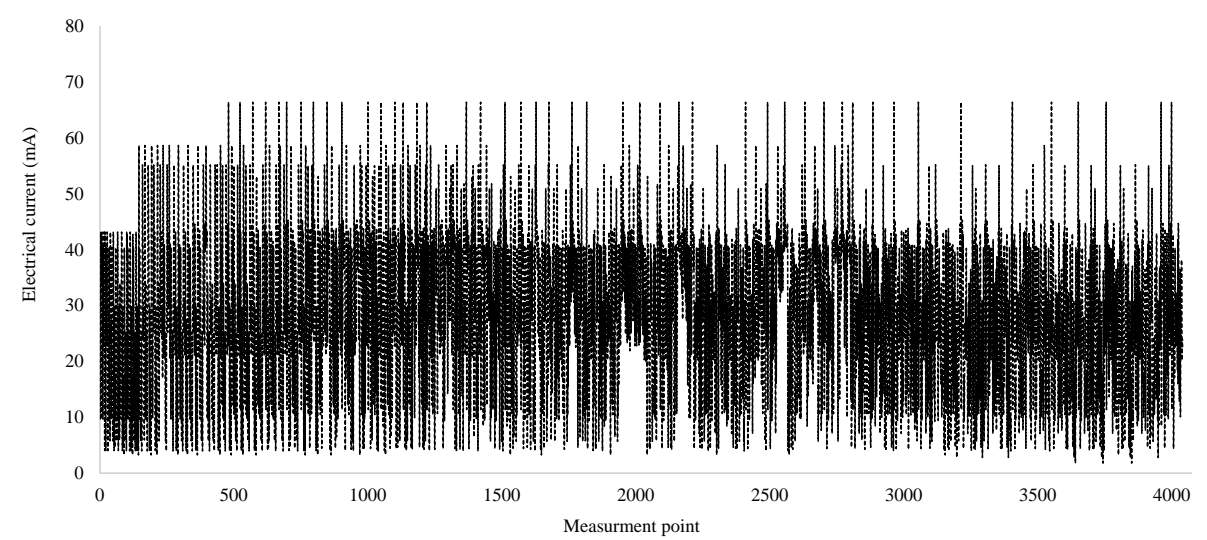

Fig. 17. The data of measurement current. 


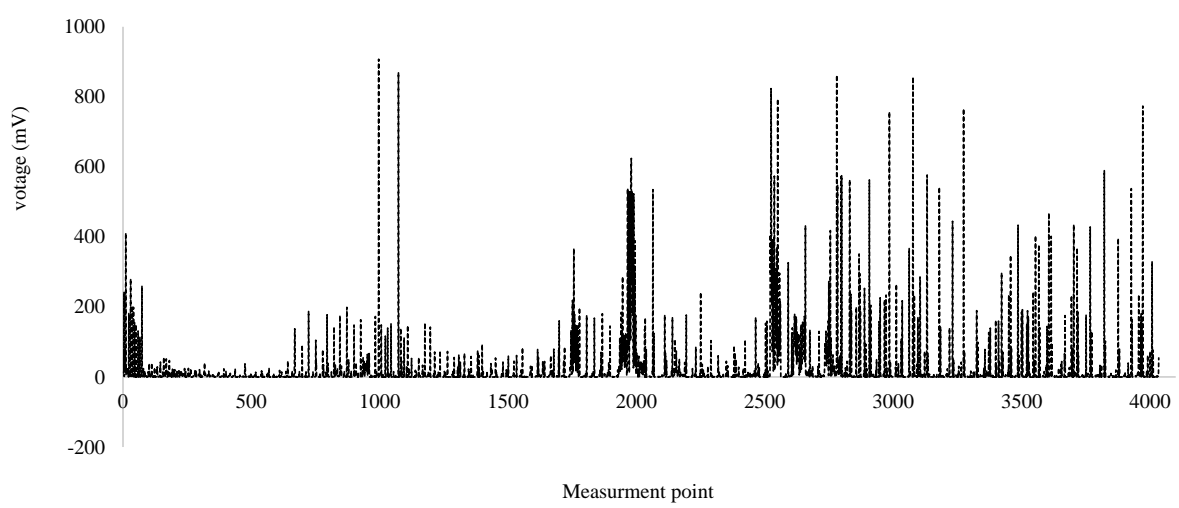

Fig. 18. The data of voltage.

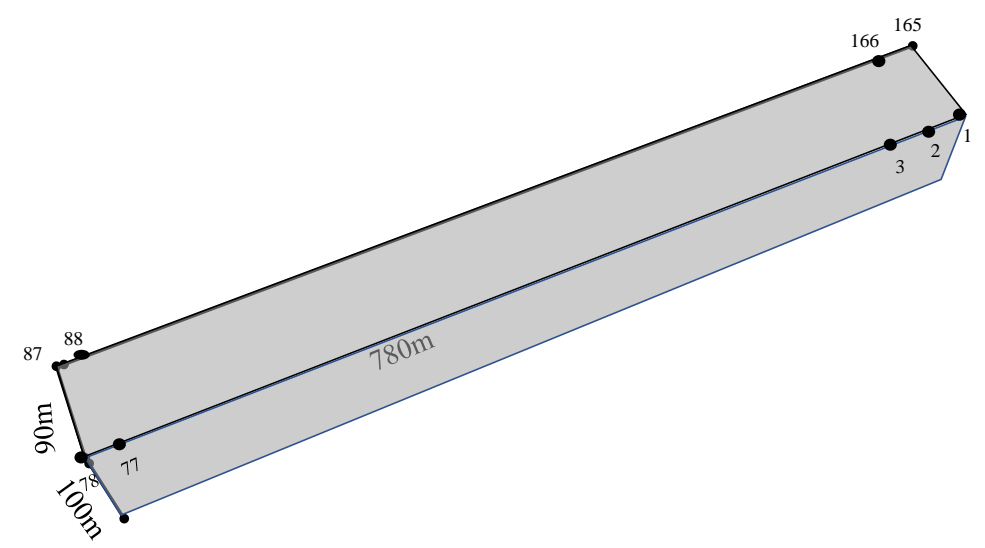

Fig. 19. The geological model of Part 2.

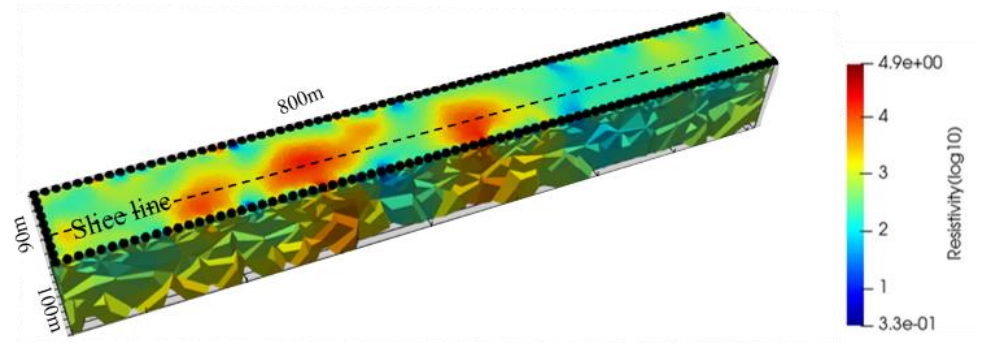

Fig. 20. Inversion results from the collected electrical potential of Part 2.

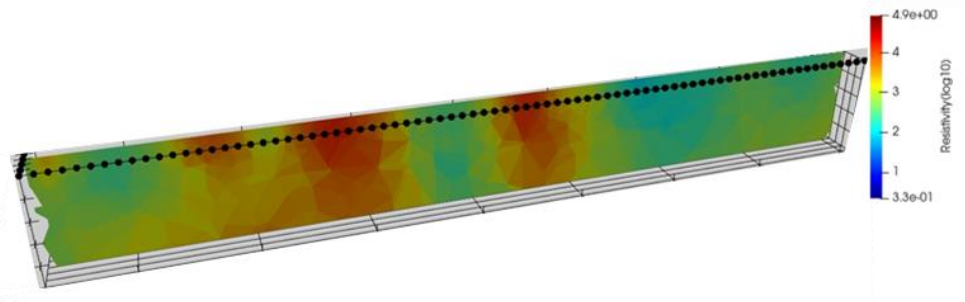

Fig. 21. The slice of the inversion results as described in Fig. 20.

After the field work, all geological anomalous information has been verified by geology drilling. Finally, these observations are consistent with the actual condition of the real coal seam in Lan County. As a result, the proposed method can correctly detect the disaster water sources, so it can be applied to practice.

\section{DISCUSSION}

For all coal mine geophysical methods, DC resistivity is the best choice for detecting mine disaster water sources. Not only it can locate the distribution of low resistivity, but also it is free from metal interference in the coal mine tunnel. Besides, according to the character of coal mine geology, in fact, we simplify the whole space to be half space in this research, hence it is necessary to do the inversion calculation in the whole space in the future research.

\section{Conclusions}

This study aims to explore water-bearing coal seam floor and exploit the relationship between the distribution of low resistivity and water faults by the direct current resistivity method. Firstly, the forward and inversion theory methods have been studied and its effectiveness have been verified by numerical simulation. Secondly, to obtain field data, we have carried out the field work of collecting data in the coal mine tunnel and obtained two sets of data. Thirdly, the 3D data 
volumes of resistivity distribution have been computed by inversion method, and with the purpose of knowing the detailed information, two slices have been cut from Part 13D data volume and Part 2 3D data volume. Finally, based on the above research results, we totally point out three threats of mine water disaster sources. The results provide important information on coal mining safety.

\section{ACKNOWLEDGMENT}

We are grateful to the anonymous reviewers of this paper.

\section{CONFLICTS OF INTEREST}

The authors declare that they have no conflicts of interest.

\section{REFERENCES}

[1] Z. Tang, S. Yang, G. Xu, and M. Sharifzadeh, "Disaster-causing mechanism and risk area classification method for composite disasters of gas explosion and coal spontaneous combustion in deep coal mining with narrow coal pillars", Process Safety and Environmental Protection, vol. 132, pp. 182-188, 2019. DOI: 10.1016/j.psep.2019.09.036.

[2] S. Zhang, W. Guo, and Y. Li, "Experimental simulation of water-inrush disaster from the floor of mine and its mechanism investigation", Arabian Journal of Geosciences, vol. 10, no. 22, p. 503, 2017. DOI: 10.1007/s12517-017-3287-3.

[3] R. Markowska, "Exposure of underground cable intrusion detection system to transient disturbances caused by nearby lightning strikes", Elektronika ir Elektrotechnika, vol. 26, no. 4, pp. 11-17, 2020. DOI: 10.5755/j01.eie.26.4.25847.

[4] A. Roy and A. Apparao, "Depth of investigation in direct current methods", Geophysics, vol. 36, no. 5, pp. 943-959, 1971. DOI: 10.1190/1.1440226.

[5] J. Yu, R. Malekian, J. Chang, and B. Su, "Modelling of whole-space transient electromagnetic responses based on FDTD and its application in the mining industry", IEEE Transactions on Industrial Informatics, vol. 13, no. 6, pp. 2974-2982, 2017. DOI: 10.1109/TII.2017.2752230.

[6] E. Wang, P. Chen, Z. Liu, Y. Liu, Z. Li, and X. Li, "Fine detection technology of gas outburst area based on direct current method in Zhuxianzhuang Coal Mine, China", Safety Science, vol. 115, pp. 12-18, 2019. DOI: 10.1016/j.ssci.2019.01.018.

[7] P. Stummer, H. Maurer, H. Horstmeyer, and A. G. Green, "Optimization of DC resistivity data acquisition: Real-time experimental design and a new multielectrode system", IEEE Transactions on Geoscience \& Remote Sensing, vol. 40, no. 12, pp. 2727-2735, 2002. DOI: 10.1109/TGRS.2002.807015.

[8] R. Cockett, L. J. Heagy, and D. W. Oldenburg, "Pixels and their neighbors: Finite volume", The Leading-Edge, vol. 35, no. 8, pp 703-706, 2016. DOI: 10.1190/tle35080703.1.
[9] J.-H. Yue, H. Zhang, H. Yang, and F. Li, "Electrical prospecting methods for advance detection: Progress, problems, and prospects in Chinese coal mines", IEEE Geoscience and Remote Sensing Magazine, vol. 7, no. 3, pp. 94-106, 2019. DOI: 10.1109/MGRS.2018.2890677.

[10] J. M. Reynolds, An Introduction to Applied and Environmental Geophysics. Wiley-Blackwell, 2011.

[11] D. S. Parasnis, Principles of Applied Geophysics. Chapman and Hall, 1979.

[12] S. Zhao and M. J. Yedlin, "Multidomain Chebyshev spectral method for 3-D dc resistivity modelling", Geophysics, vol. 61, no. 6, pp. 1616-1623, 1996. DOI: 10.1190/1.1444080.

[13] Y. Sasaki, "3-D resistivity inversion using the finite-element method", Geophysics, vol. 59, no. 12, pp. 1839-1848, 1994. DOI: 10.1190/1.1443571.

[14] C. Rucker, T. Gunther, and K. Spitzer, "Three-dimensional modelling and inversion of dc resistivity data incorporating topography - I. Modelling", Geophysical Journal International, vol. 166, no. 2, pp. 495-505, 2006. DOI: 10.1111/j.1365-246X.2006.03010.x.

[15] G. A. Newman, "A Review of high-performance computational strategies for modeling and imaging of electromagnetic induction data", Surveys in Geophysics, vol. 35, no. 1, pp. 85-100, 2014. DOI: 10.1007/s10712-013-9260-0.

[16] Y. Li, R. Brossier, and L. Métivier, "3D frequency-domain elastic wave modeling with spectral-element method using a massively parallel direct solver", Geophysics, vol. 85, no. 2, pp. T71-T88, 2020. DOI: 10.1190/geo2019-0172.1.

[17] J. Wu, "My humble opinions on Shanxi's coal resource advantage turning into economic advantage", Coal Economic Research, no. 7, pp 12-14, 1995. DOI: CNKI:SUN:MTJN.0.1995-07-003(In Chinese).

[18] F. G. Bell, Environmental Geology: Principles and Practice. Oxford: Blackwell, 1998.

[19] V. Puzyrev, S. Koric, and S. Wilkin, "Evaluation of parallel direct sparse linear solvers in electromagnetic geophysical problems", Computers \& Geosciences, no. 89, pp. 79-87, 2016. DOI: 10.1016/j.cageo.2016.01.009.

[20] I. Priezzhev and H. Pfutzner, "Method for 3-D gravity forward modeling and inversion in the wave number domain", U.S. Patent, 20120232871 A1, 2014. [Online]. Available: http://www.google.com/patents/US8700372

[21] R. Cockett, "Direct current resistivity inversion using various objective functions (Unpublished work style)", unpublished.

[22] H. Mizunaga and K. Ushijima, "Three-dimensional numerical modeling for Mise-ó-la-masse method", BUTSURI-TANSA (Geophysical Exploration), vol. 44, no. 4, pp. 215-226, 1991.

[23] B. Zehner, J. H. Borner, I. Gorz, and K. Spitzer, "Workflows for generating tetrahedral meshes for finite element simulations on complex geological structures", Computers \& Geosciences, vol. 79, pp. 105-117, 2015. DOI: 10.1016/j.cageo.2015.02.009.

[24] A. James, B. Geveci, and C. Law, ParaView: An End-User Tool for Large Data Visualization, Visualization Handbook, Elsevier, 2005.

[25] P. Kearey, M. Brooks, and I. Hill, An Introduction to Geophysical Exploration. Wiley-Blackwell, 2002, ch. 8.

This article is an open access article distributed under the terms and conditions of the Creative Commons Attribution 4.0 (CC BY 4.0) license (http://creativecommons.org/licenses/by/4.0/). 